\title{
Improvement of Liver Transplantation Outcome by Heme Oxygenase-1-Transduced Bone Marrow Mesenchymal Stem Cells in Rats
}

\author{
Bin Wu, ${ }^{1}$ Hong-Li Song, ${ }^{1,2}$ Yang Yang, ${ }^{3}$ Ming-Li Yin, ${ }^{3}$ Bo-Ya Zhang, ${ }^{3}$ Yi Cao, ${ }^{3}$ \\ Chong Dong, ${ }^{1}$ and Zhong-Yang Shen ${ }^{1}$ \\ ${ }^{1}$ Department of Organ Transplantation, Tianjin First Central Hospital, Tianjin 300192, China \\ ${ }^{2}$ Tianjin Key Laboratory of Organ Transplantation, Tianjin 300192, China \\ ${ }^{3}$ Tianjin First Central Hospital Clinic Institute, Tianjin Medical University, Tianjin 300070, China
}

Correspondence should be addressed to Hong-Li Song; hlsong26@163.com and Zhong-Yang Shen; shenzhy@tmu.edu.cn

Received 21 September 2015; Revised 30 November 2015; Accepted 1 December 2015

Academic Editor: Laura Lasagni

Copyright (C) 2016 Bin Wu et al. This is an open access article distributed under the Creative Commons Attribution License, which permits unrestricted use, distribution, and reproduction in any medium, provided the original work is properly cited.

\begin{abstract}
Bone marrow mesenchymal stem cells (BMMSCs) exert immunosuppressive activity in transplantation, and heme oxygenase-1 (HO-1) enhances their immunomodulatory effects. The aim of this study was to determine whether HO-1-transduced BMMSCs (HO-1/MSCs) improve rat liver transplantation (LTx) outcomes. Orthotopic LTx rejection models were treated with HO-1/MSCs, BMMSCs, HO-1, or normal saline, respectively. Our results showed a significant improvement in survival rates in the HO1/BMMSCs group compared to the control groups. At all time points, liver function marker levels in the HO-1/MSCs group were significantly lower than in the other three groups; on POD 1, 7, and 14, the degree of rejection and apoptotic cells was significantly less in the HO-1/MSCs group than in the other three groups. Interleukin- (IL-) 10 and transforming growth factor- $\beta$ levels were significantly increased, while IL-2, IL-6, IL-17, IL-23, tumor necrosis factor- $\alpha$, and interferon- $\gamma$ levels were significantly decreased in the HO-1/MSCs group when compared to the other groups. Splenocyte Tregs were significantly increased by HO-1/MSCs compared with controls on POD 3, 5, 7, 10,14, and 28. Summarily, we provide evidence that HO-1/MSCs improved allogeneic LTx outcomes by attenuating inflammatory responses and acute cellular rejection, as well as enhanced immunomodulatory effects compared with BMMSCs.
\end{abstract}

\section{Introduction}

Liver transplantation (LTx) is currently the only effective treatment for end-stage liver diseases, such as acute or chronic liver failure. However, the shortage of donor organs and issues of rejection and adverse reactions from immunosuppressants have hindered the use of Ltx. Immune rejection and ischemia-reperfusion injury after transplantation are two main reasons for loss of the graft [1]. Therefore, approaches to minimize rejection and induction of immune tolerance may be used to effectively address the above problems [2]. Clinical treatment of acute rejection (ACR) after LTx requires high doses of immunosuppressants. However, longterm use of immunosuppressive agents raises the risk of unfavorable side effects, such as nephrotoxicity, neurotoxicity, hypertension, posttransplant malignancy, and metabolic deterioration. Thus, safer and more effective strategies to attain immunosuppression are urgently needed.

Bone marrow mesenchymal stem cells (BMMSCs) are currently investigated in studies focused on transplantation immunity [3-5]. Because BMMSCs possess not only multidirectional differentiation potential and self-renewal capacity but also immunomodulatory activities, they are valuable in applications such as organ transplantation [6]. As a population of pluripotent stem cells from bone marrow, BMMSCs can be easily obtained and separated, as well as being cultivated and amplified in vitro. They may also be used in vivo without rejection and ethical obstacles. Based on the reasons above, we chose to use BMMSCs in our work. In recent years, BMMSCs have been shown to have low immunogenicity and therefore can suppress $\mathrm{T}$ cell-mediated immune rejection following organ transplantation [7], as 
well as participating in immunosuppression by influencing cytokine secretion from $\mathrm{T}$ cells and interacting with antigen presenting cells such as dendritic cells [8]. However, purified BMMSCs have been reported to have low activity after infusion in vivo [9], which was also noted in our earlier work.

Heme oxygenase $(\mathrm{HO})$ is the rate-limiting enzyme in the degradation of heme to biliverdin and subsequently to bilirubin [10]. HO-1, an inducible isoform of $\mathrm{HO}$, is a potent cytoprotective factor that has been shown to have antiinflammatory, anti-ischemia-reperfusion injury, and antiapoptotic properties [11-13]. As an active regulatory factor involved in the control of immune tolerance after organ transplantation [14], HO-1 can increase the immunomodulatory effects of $\mathrm{T}$ regulatory cells (Tregs) by promoting the secretion of interleukin- (IL-) 10 and transforming growth factor- (TGF-) $\beta$ to increase tolerance to grafts in recipients [15]. HO-1 also has been shown to reduce the apoptosis of BMMSCs under conditions of hypoxia and oxidative stress [16]. At present, genetic engineering can be used to effectively modify protein expression of BMMSCs [17]. Recent studies have found that transduction of HO-1 into BMMSCs improved their transformation ability [18], affected their immunomodulatory effects [19] and antioxidant capacity, and enhanced the strength and durability of their activities. In our previous studies, we found that HO-1-transduced BMMSCs (HO-1/MSCs) had improved immunoregulatory effects on lymphocytes in vitro compared with unmodified BMMSCs [20]. Thus, in this study, we verified those in vitro results using an orthotopic LTx rejection model to further determine whether HO-1/MSCs can improve outcomes of LTx in rats.

\section{Materials and Methods}

2.1. Animals and Ethics. Specific pathogen-free (SPF) experimental animals were obtained from the Vital River Company (Beijing, China). Inbred adult male Lewis rats (220$250 \mathrm{~g}, 8-10$ weeks old) were LTx donors, and inbred adult male Brown Norway (BN) rats (220-250 g, 8-10 weeks old) were recipients. BMMSCs were extracted from inbred adult male BN rats (100-120 g, 4-5 weeks old). Before testing, all rats were housed individually for 3 days in standard animal facilities on a $12 \mathrm{~h}$ light/dark cycle and provided with commercially available chow and tap water ad libitum. All experimental procedures were carried out in accordance with the Guide for the Care and Use of Laboratory Animals of the National Institutes of Health (NIH publication 86-23, revised 1985). All protocols were approved by the Animal Care and Research Committee of Tianjin First Central Hospital, Tianjin, China (Permit number: E20130825-003A). Surgeries were performed using chloral hydrate anesthesia, and all efforts were made to minimize animal suffering.

2.2. Isolation and Characterization of BMMSCs. Using the method described by Pittenger et al. [21], BMMSCs were isolated from the femur and tibia of male $\mathrm{BN}$ rats (100$120 \mathrm{~g}, 4-5$ weeks old) after sacrifice by cervical dislocation. After lysis of red blood cells using $0.1 \mathrm{~mol} / \mathrm{L} \mathrm{NH}_{4} \mathrm{Cl}$, the remaining cells were washed, resuspended, and cultured for 4 weeks at $37^{\circ} \mathrm{C}$ with $5 \% \mathrm{CO}_{2}$ in Dulbecco's modified Eagle medium (DMEM)/F12 (Gibco, Carlsbad, CA, USA) containing $100 \mathrm{U} / \mathrm{mL}$ penicillin, $100 \mathrm{mg} / \mathrm{mL}$ streptomycin, and $15 \%$ fetal bovine serum. The culture medium was changed every $72 \mathrm{~h}$. After reaching $80 \%$ confluency, cells at the third passage were trypsinized, washed, centrifuged, and resuspended at $1 \times$ $10^{7}$ cells $/ \mathrm{mL}$ in phosphate-buffered saline (PBS). BMMSCs were labeled with antibodies against CD29, CD90, CD34, CD45, RT1A, and RT1B (Santa Cruz Biotechnology, Dallas, TX, USA) for flow cytometric analysis (BD FACSAria III, Franklin Lakes, NJ, USA). BMMSCs were also confirmed by light microscopy to be adherent to plastic and spindleshaped.

2.3. Transduction of BMMSCs with HO-1-Bearing Recombinant Adenovirus. Adenovirus/HO-1 (HO-1) obtained from Shanghai Genechem Limited Company (Shanghai, China) was diluted to $10 \mathrm{pfu} / \mathrm{cell}$ with complete culture solution and used to replace the original medium of the BMMSC cultures. After 6 to $8 \mathrm{~h}$, the HO-1 culture solution was exchanged with complete culture solution for continued cultivation of the BMMSCs. After $48 \mathrm{~h}$, the infection efficiency was observed under a fluorescence microscope. Molecular biological features of HO-1/MSCs were assessed by flow cytometry.

2.4. Surgical Procedures and Experimental Protocol. An orthotopic LTx rejection model was performed with Lewis donor rats and $\mathrm{BN}$ recipient rats in a sterile field under general anesthesia using $5 \%$ chloral hydrate $(10 \mathrm{~mL} / \mathrm{kg})$. Food was withheld from both donor and recipient animals for $12 \mathrm{~h}$ prior to surgery, and differences between their weights were not greater than $10 \mathrm{~g}$. The two-cuff technique for LTx was used by a single operator [22]. The donor livers were perfused via the portal vein (PV) with $4^{\circ} \mathrm{C}$ lactated Ringer's solution containing heparin sodium $(50 \mathrm{U} / \mathrm{mL})$. The harvested graft was preserved in a bath of lactated Ringer's solution at $4^{\circ} \mathrm{C}$. The cuff was slipped over the PV using microforceps, and the distal end of the vein was everted over the cuff and secured with a circumferential 5-0 silk ligature. The same procedure was performed for the infrahepatic vena cava (IHVC) cuff preparation. After the recipient liver was removed, the donor liver was placed orthotopically in the abdominal cavity of the recipient. The suprahepatic vena cava (SHVC) was anastomosed end to end using a continuous 7-0 nylon suture. The cuff anastomosis of the PV and IHVC was then performed. The graft was reperfused by opening the PV, IHVC, and SHVC in turn. The bile duct was connected by telescoping a tube in the bile duct of the donor into that of the recipient. Experimental animals were divided into four groups, which received HO-1/MSCs, BMMSCs, $\mathrm{HO}-1$, or normal saline. Rats of the treatment group received $5 \times 10^{6} \mathrm{HO}-1 / \mathrm{MSCs}$ through the superficial dorsal veins of the penis immediately after the surgery, while the control groups were given $5 \times$ $10^{6}$ BMMSCs, $5 \times 10^{7} \mathrm{HO}-1$, or the equivalent volume of normal saline. Five animals per group were euthanized on postoperative day (POD) 1, 3, 5, 7, 10, 14, or 28 .

2.5. Survival Rates of Recipients. Recipient survival rates and clinical manifestations were observed in five animals per 
group. Humane endpoints were used for moribund animals after surgery, especially in the survival study. All animals meeting the humane endpoint criteria were euthanized by intraperitoneal injection of excess chloral hydrate when there was no unexpected death.

2.6. Liver Function Assay. Recipient serum was obtained from peripheral blood. Levels of alanine transaminase (ALT), aspartate aminotransferase (AST), and total bilirubin (TBIL) were measured using an automatic biochemical analyzer (Olympus Au640, Tokyo, Japan).

2.7. Histopathological Analysis. After fixation in $10 \%$ formalin, hepatic tissues were embedded in paraffin and cut into $5 \mu \mathrm{m}$ thick sections, followed by staining with hematoxylin and eosin (H\&E). Pathological changes and the extent of rejection were evaluated under a light microscope. ACR was classified according to the Banff criteria [23].

2.8. Apoptosis Assay. Terminal deoxynucleotidyl transferase(TdT-) mediated dUTP nick end-labeling (TUNEL) staining was performed on paraffin-embedded tissue sections using the In Situ Cell Death Detection Kit (Roche Biochemicals, Mannheim, Germany), as instructed by the manufacturer. Tdt was not used in the negative controls, and deoxyribonuclease was used for the positive controls. Apoptotic nuclei would appear brown, while the staining of the cytoplasm would generally be light or absent. The slides were reviewed in a blinded fashion, and positive cells were counted in 10 randomly chosen fields under a light microscope (200x).

2.9. Enzyme-Linked Immunosorbent Assay (ELISA). Serum was obtained from peripheral blood of recipients. Using ELISA, serum concentrations of cytokines related to inflammatory responses, T helper (Th)1/Th2, and Th17/Tregs were assayed at the same time points after LTx. Interleukin(IL-) 10, TGF- $\beta$, IL-2, IL-6, IL-17, IL-23, tumor necrosis factor- (TNF-) $\alpha$, and interferon- (IFN-) $\gamma$ concentrations were measured using ELISA kits (Santa Cruz Biotechnology) according to the manufacturer's instructions.

2.10. Flow Cytometry. Lymphocytes were isolated from recipient spleens. Aliquots of $1 \times 10^{7}$ cells were resuspended in $0.1 \mathrm{~mL}$ PBS and labeled with antibodies specific for CD4, CD25, and Foxp3 (eBioscience, San Diego, CA, USA) or their isotype-matched control antibodies for analysis by flow cytometry (BD FACSAria III). The anti-CD4 antibody was conjugated to fluorescein isothiocyanate (FITC), and the isotype-matched antibody was mouse immunoglobulin $G$ (IgG)2a K-FITC. The anti-CD25 antibody was conjugated to phycoerythrin (PE), and the isotype-matched antibody was mouse IgG1 K-PE. The anti-Foxp3 antibody was conjugated to PerCP-Cyanine5.5, and the isotype-matched antibody was rat IgG2a K-PerCP-Cyanine5.5.

2.11. Statistical Analysis. Results are expressed as the mean \pm standard deviation (SD) and compared by one-way analysis of variance. Kaplan-Meier analysis was used to compare survival rates of recipients, and log-rank testing was used to determine significant differences between groups. All statistical analyses were performed using SPSS statistical software, version 17.0 (SPSS GmbH, Munich, Germany), with $P \leq 0.05$ considered statistically significant.

\section{Results}

3.1. Culture and Identification of BMMSCs. Cells isolated from rat bone marrow were confirmed as BMMSCs based on their spindle-shaped morphology, adherence to plastic, and phenotypic characterization by flow cytometry (Figure 1(a)). Surface marker detection of Ad/HO-1/BMMSCs showed that they were positive for CD29, CD90, and RT1A and negative for CD34, CD45, and RT1B (Figures 1(d), 1(e), and 1(f)).

3.2. Generation of HO-1/MSCs. At $48 \mathrm{~h}$ after HO-1 transduction, BMMSCs were observed by fluorescence microscopy, which showed that the infection efficiency was about $85 \%$ (Figures 1(b) and 1(c)) and indicated that HO-1/MSCs were generated successfully.

3.3. Improvement of Clinical Manifestations and Recipient Survival Rates. From POD 3 to 5, recipients in the HO1 and normal saline groups had poor appetite, decreased activity, untidy and lusterless hair, jaundice, and lethargy. Most of them died within one month. However, most of the recipients in groups treated with $\mathrm{HO}-1 / \mathrm{MSC}$ and BMMSCs survived for more than two months and were more active and responded more quickly to stimulation. The median recipient survival times were $77,61,25$, and 21 days in the HO-1/MSCs, BMMSCs, HO-1, and normal saline groups, respectively. The survival time of the HO-1/MSCs group was significantly different from that of the other groups $(P<0.05)$, and that of the BMMSCs group was significantly different from the HO1 and normal saline groups (61 versus 25 versus 21 days, $P<$ 0.05 ; Figure $2(\mathrm{a})$ ). Thus, HO-1/MSCs significantly improved the recipient survival rates.

3.4. Serum Levels of Markers of Recipient Liver Function. Within 7 days of LTx, levels of ALT and AST in all groups decreased at first, then increased, and finally decreased again. The level of TBIL in the normal saline and HO-1 groups increased steadily between POD 5 and 7, increased sharply on POD 10, and then decreased significantly but remained at a relatively high level. During that time, TBIL in the HO-1/MSCs and BMMSCs groups increased steadily to the maximum on POD 28, except for a slight decrease on POD 7. At all time points liver function marker levels in the HO$1 /$ MSCs group were lower than in the other three groups $(P<$ 0.05); those in the BMMSCs group were also significantly different when compared to the HO-1 and normal saline groups $(P<0.05$; Figure 2(b)).

3.5. Histopathological Analysis and Grading of ACR in Hepatic Grafts. ACR was classified by the grade of inflammatory infiltrate in the portal space, around biliary ducts and in vessel walls [19]. To analyze the effects of treatment with normal saline, HO-1, BMMSCs, and HO-1/MSCs on ACR of hepatic 


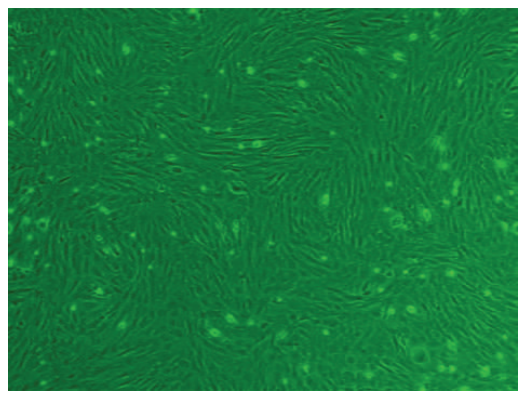

(a)

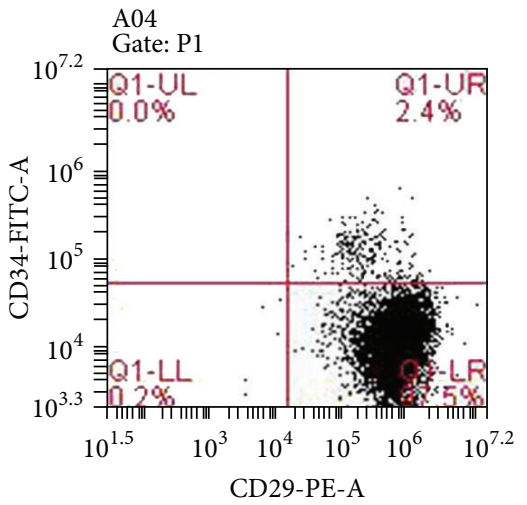

(d)

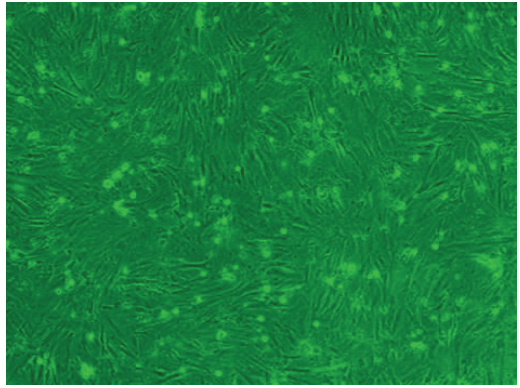

(b)

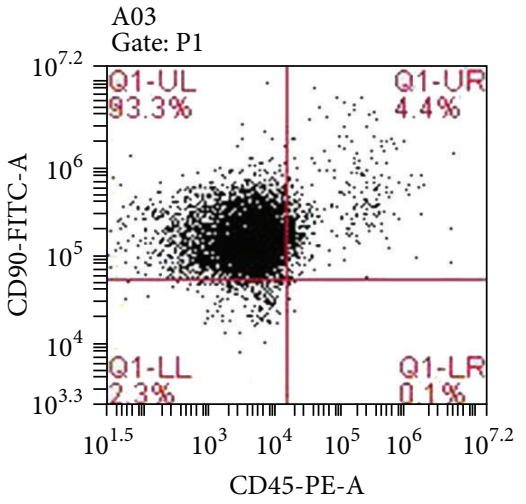

(e)

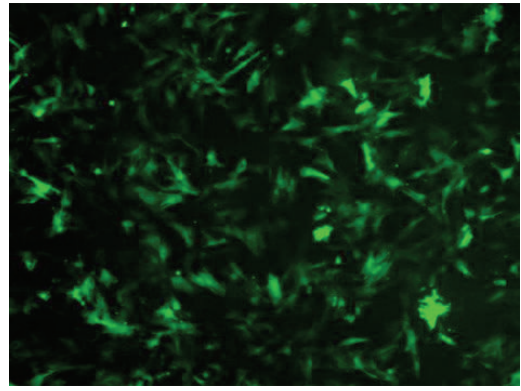

(c)

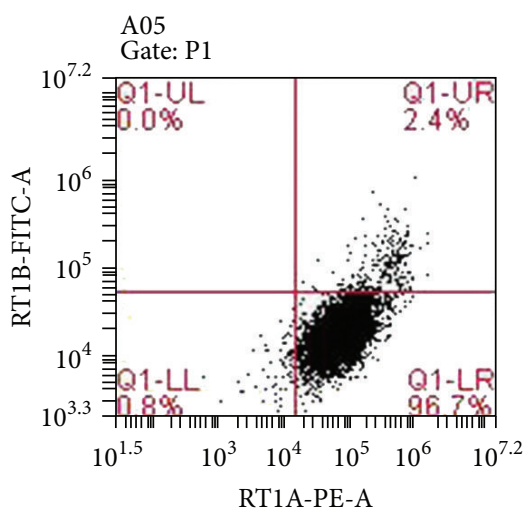

(f)

Figure 1: Morphological (100x) and flow cytometric analysis of BMMSCs. (a) Morphology of third-passage BMMSCs. (b) Morphology of BMMSCs after transduction with HO-1 in a bright field. BMMSCs exhibited spindle-shaped morphology and were arranged in whorls when transfected with HO-1. (c) Morphology of BMMSCs after transduction with HO-1 in a fluorescent field. Over 85\% of BMMSCs after transduction with HO-1 emitted green fluorescence. (d) The proportion of CD29-positive and CD34-negative cells was 97.5\%. (e) The proportion of CD90-positive and CD45-negative cells was 93.3\%. (f) The proportion of RT1A-positive and RT1B-negative cells was 96.7\%.

grafts in allogeneically transplanted animals, we assessed the histopathological grade of ACR at each time point after LTx. The animals treated with normal saline and HO-1 showed a gradual increase in acute rejection, with moderate or severe acute rejection observed on POD 5 and 7. Animals in both the BMMSCs and HO-1/MSCs treatment group showed reduced ACR to different degrees. The pathology of the HO-1/MSCs treatment group was characterized by mild rejection within POD 7, and the degree of rejection increased progressively thereafter; however, the degree of rejection in the HO-1/MSCs group was less than in the other three groups $(P<0.05)$, and that in the BMMSCs group was less than in the HO-1 and normal saline groups, at all time points $(P<0.05$; Figure 3$)$.

3.6. Evaluation of Apoptosis. ACR is associated with increased apoptosis in the graft. Using TUNEL staining, we evaluated apoptotic cells in the hepatic tissue after LTx (Figure 4(a)). On POD 1, 7, and 14, there were fewer apoptotic cells in the HO-1/MSCs group than in the other three groups $(P<0.05)$, and there were fewer apoptotic cells in the BMMSCs group than in the normal saline and HO-1 groups $(P<0.05)$. Treatment with $\mathrm{HO}-1 / \mathrm{MSC}$ significantly reduced TUNEL-positive apoptotic cells in hepatic tissue at each time point (Figure 4(b)).
3.7. Recipient Serum Cytokine Concentrations. ELISAs were performed to assay the serum concentrations of cytokines related to inflammatory responses and differentiation of $\mathrm{T}$ cells, such as Th1/Th2 and Th17/Tregs (Figure 5). The HO1 and normal saline treatment groups showed rising trends in concentrations of IL-2, TNF- $\alpha$, IFN- $\gamma$, IL- 6 , IL-17, and IL23 , and these levels were significantly increased compared with the BMMSCs and HO-1/MSCs group. Serum IL-10 and TGF- $\beta$ concentrations in both the HO-1 and normal saline treatment groups also decreased rapidly. The decreases in IL2, IL-6, and IL-17 levels were much lower in the BMMSCs treatment group compared with the allogeneic control group; however, their concentrations sharply increased on POD 10 compared with other time points. Concentrations of IL-23, TNF- $\alpha$, and IFN- $\gamma$ exhibited a relative small decrease in the BMMSCs treatment group, while those of IL-10 and TGF$\beta$ continuously increased. As for the HO-1/MSCs treatment group, IL-23, TNF- $\alpha$, and IFN- $\gamma$ exhibited relatively small decreases in concentration, and levels of IL-6, IL-17, and IL-2 demonstrated a downward trend until POD 10 and an upward trend thereafter; meanwhile, IL-10 and TGF- $\beta$ concentrations continuously increased at all time points.

3.8. Proportion of Recipient Spleen Tregs. Using flow cytometry, CD4+CD25+Foxp3+Tregs were assayed at various time 


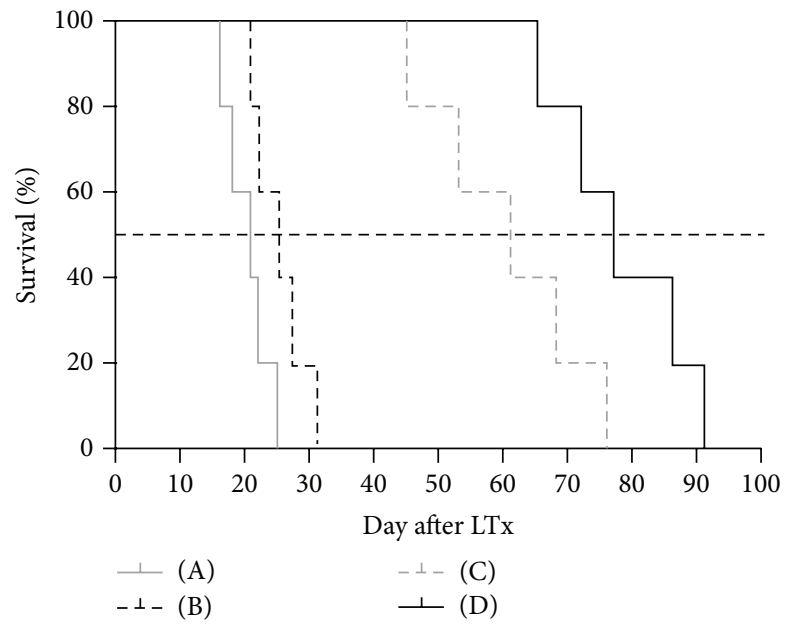

(a)
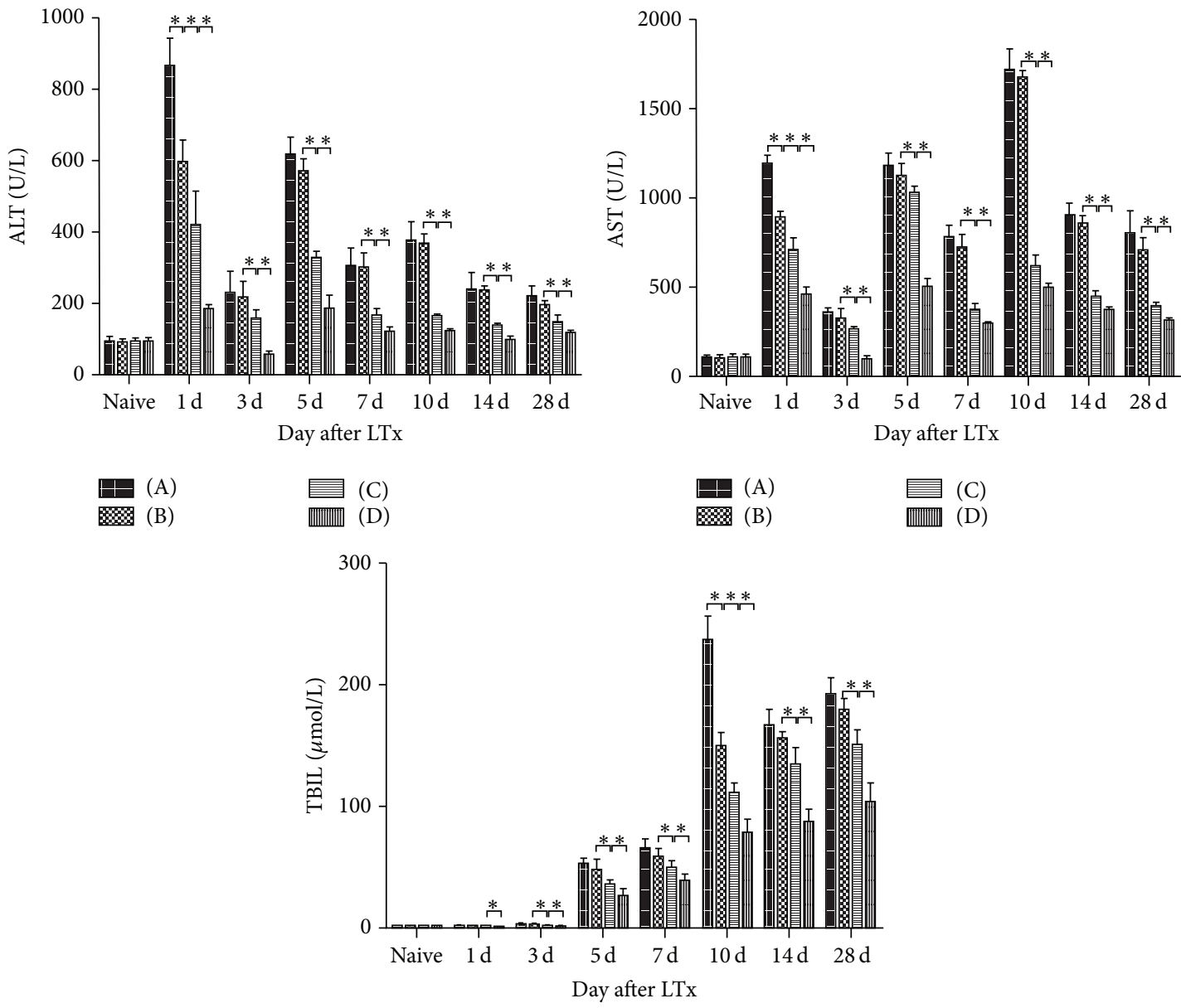

(A)

(B)

(C)

血血（D)

(b)

Figure 2: Kaplan-Meier survival curve of recipients. (A) Normal saline group; (B) HO-1 group; (C) BMMSCs group; (D) HO-1/MSCs group. (a) Median recipient survival times were 77, 61, 25, and 21 days in the HO-1/MSCs group, BMMSCs group, HO-1, and normal saline groups, respectively ( $n=5$ per group). Survival rates were significantly improved in the HO-1/MSCs treatment group compared with the three control groups $(P<0.05)$. (b) Levels of ALT, AST, and TBIL were significantly lower in the HO-1/MSCs group compared with control groups at all time points $\left({ }^{*} P<0.05\right)$. 
(A)
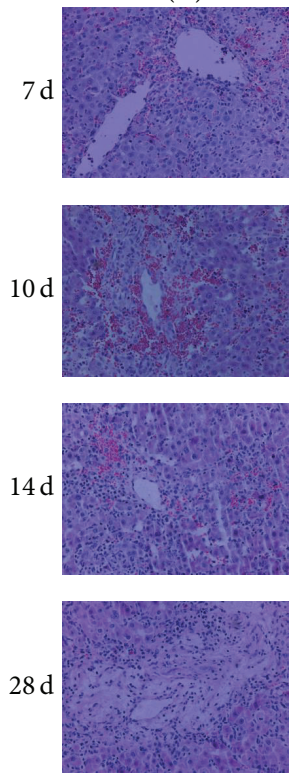

(B)
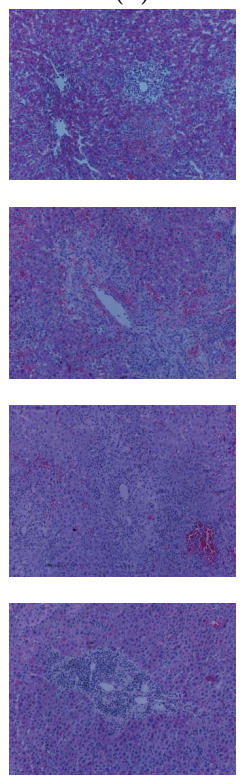

(C)
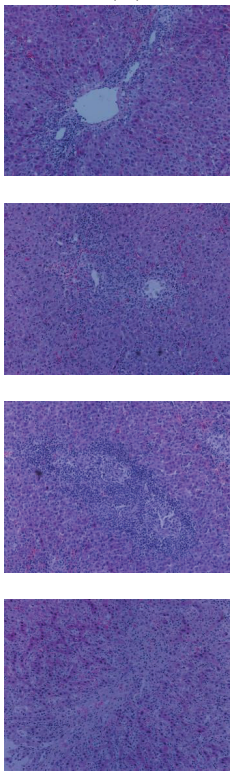
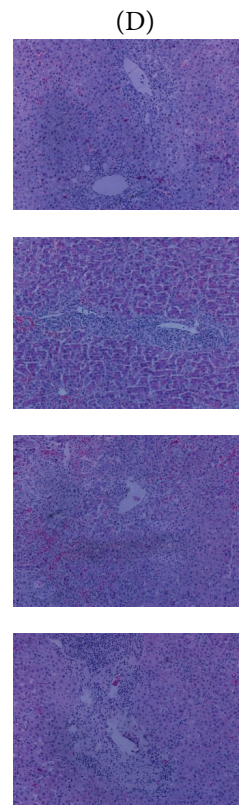

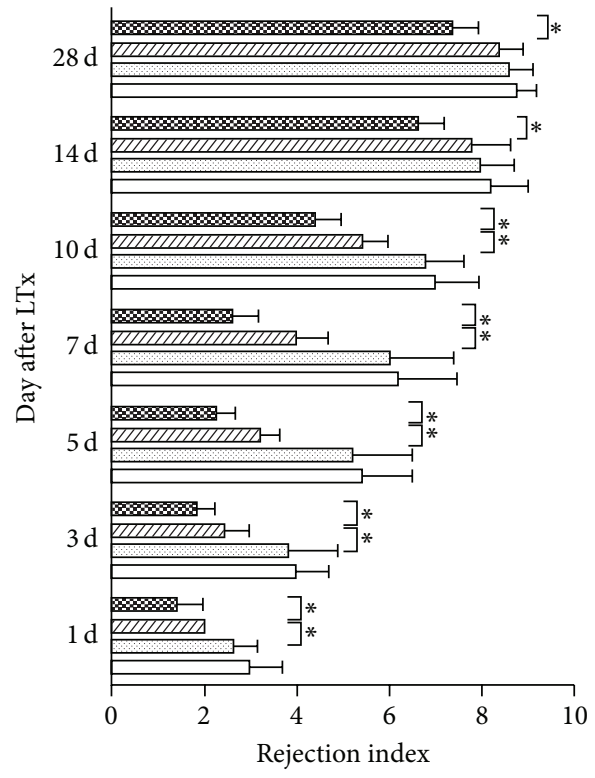

(A)

(B)

(a)

(b)

FIGURE 3: Histological sections of the rat liver and grading of ACR after LTx. (A) Normal saline group; (B) HO-1 group; (C) BMMSCs group; (D) HO-1/MSCs group. (H\&E staining, 100x). (a) In the normal saline and HO-1 groups, ACR grading on day 7 was moderate to severe, with abundant mixed lymphocytes in the portal area, interlobular bile duct inflammation and damage, inflammatory cell infiltration in the vein area, and necrosis of liver cells. In the BMMSCs group, ACR grading was mild until day 7, was aggravated sharply after day 7, and was moderate to severe on day 14. ACR was attenuated in the HO-1/MSCs group and decreased at all time points, compared to control groups $\left({ }^{*} P<0.05\right)$. (b) Histogram of ACR grading ( $n=5$ in each group, the HO-1-transduced BMMSCs group compared to control groups). Data are expressed as the mean $\pm \mathrm{SD}\left({ }^{*} P<0.05\right)$.

points after LTx (Figure 6). The proportion of Tregs in splenocytes in the normal saline and $\mathrm{HO}-1$ groups gradually decreased over time, with no significant difference between them. Levels of Tregs in the BMMSCs group first increased and then decreased, reaching the peak on POD 7 and showing significantly higher percentages than those of the normal saline group on POD $5,7,10,14$, and $28(P<0.05)$. Levels of Tregs in the HO-1/MSCs group also first increased and then decreased; however, they peaked on POD 14 and showed significantly higher proportions than those of other groups on POD $3,5,7,10,14$, and $28(P<0.05)$.

\section{Discussion}

LTx is an effective treatment for end-stage liver diseases, but rejection and ischemia-reperfusion injury can affect survival in LTx recipients and mainly account for transplantation failures. Although the use of immunosuppressive drugs can mitigate immune rejection, their long-term application can also result in high costs and side effects, such as immune deficiency and tumor susceptibility. Induction of tolerance, a long-term and specific state of anergy of the immune system, in graft recipients should be achieved while maintaining their ability to respond to other foreign antigens. Thus, inducing a lasting and stable tolerance without drugs is an ideal goal [24]. Research studies worldwide have shown that BMMSCs and HO-1 not only exert independent immune protective effects in LTx but HO-1 also can help BMMSCs to protect grafts $[12,13,25]$. Therefore, this study was designed to observe the immune regulatory effects of HO-1/MSCs in LTx and explore the potential related mechanism.

In the current study, an orthotopic liver LTx rejection model was established with major histocompatibility complex- (MHC-) disparate rat strains (Lewis to $\mathrm{BN}$ ). Histopathological analyses showed that the ACR reaction gradually increased in the normal saline group, with moderate to severe ACR occurring on day 7. The trend in the HO-1 group was nearly the same as that in the normal saline group, in which the degree of ACR was slight and not obviously different, suggesting that the effect of infusing pure HO-1 was not ideal. BMMSCs could attenuate the allograft rejection until day 7 . From day 10 , however, the improvement was attenuated, likely due to the reduction in numbers of BMMSCs and their diminished effects in vivo. Not only did HO-1/MSCs markedly attenuate the allograft rejection, but the improvement was still obvious after day 7 , indicating that HO-1 could enhance and prolong the duration of the immune activities of BMMSCs in vivo, thus resulting in better 
(A)

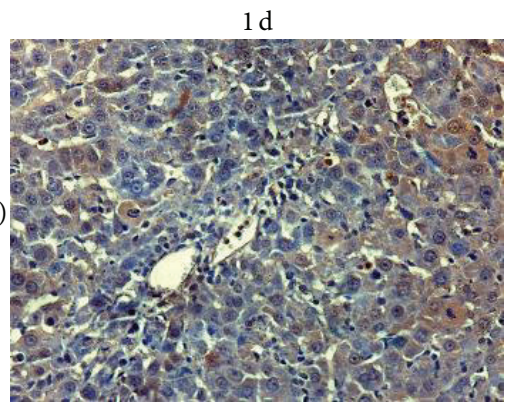

(B)

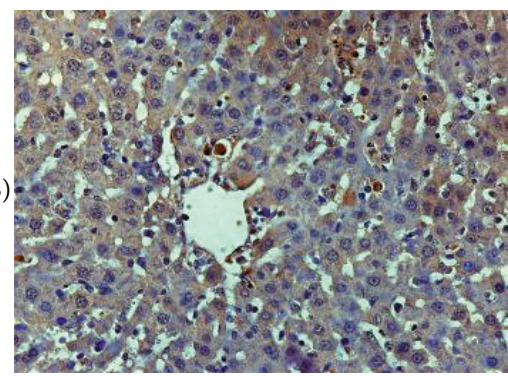

(C)

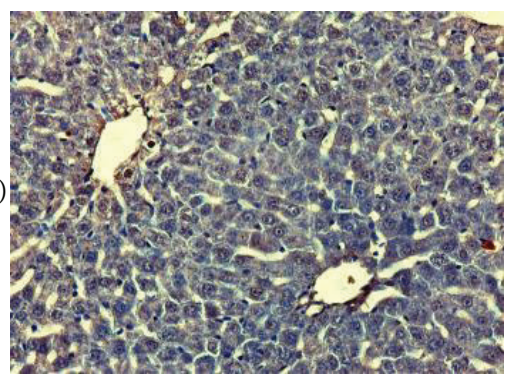

(D)
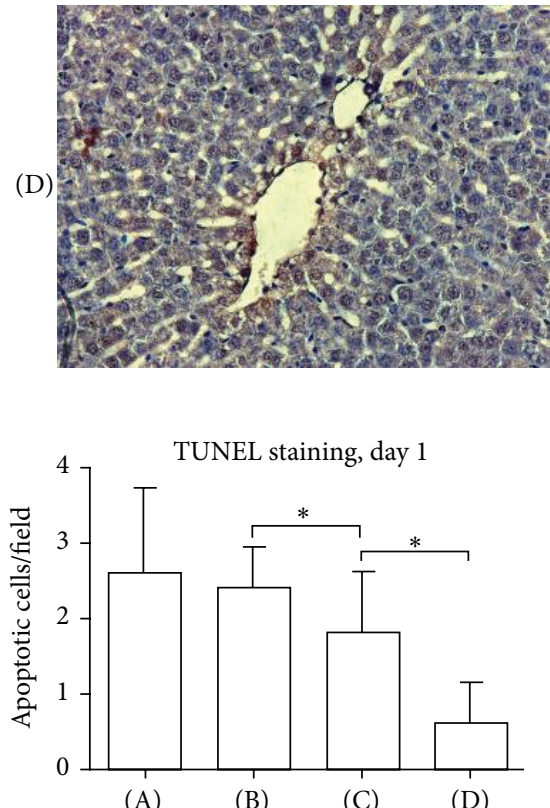
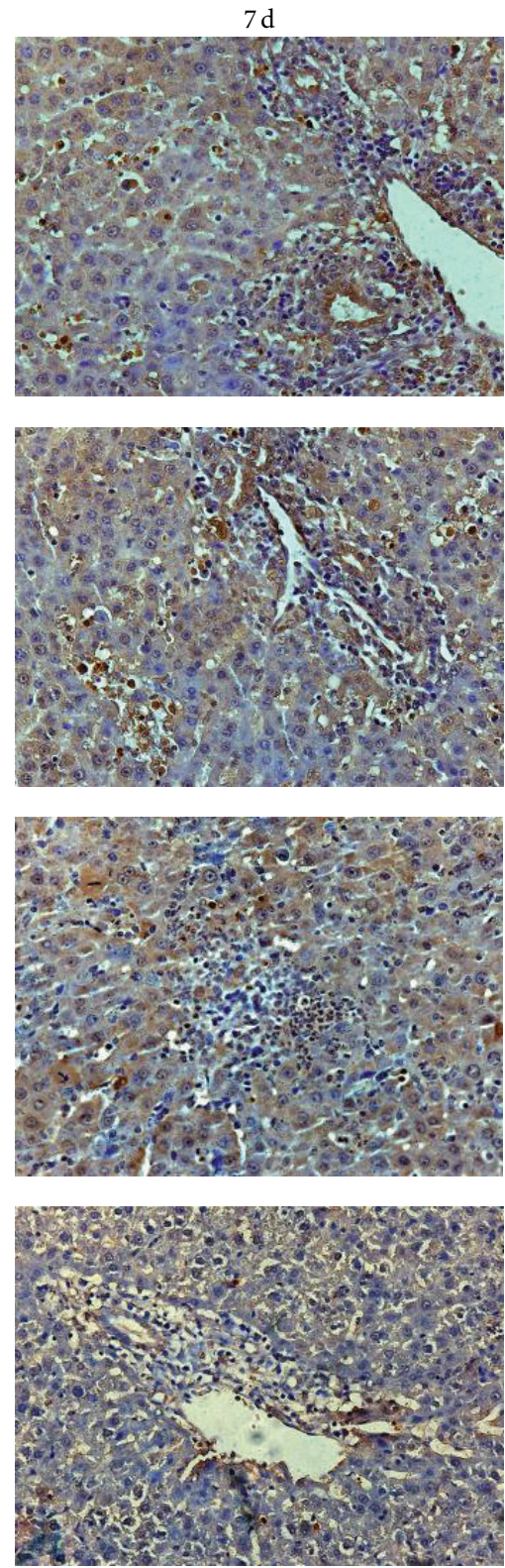

(a)

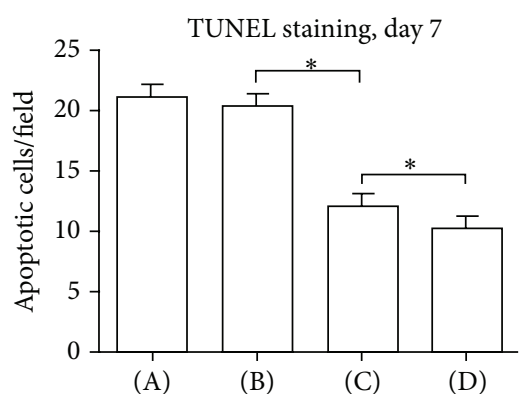

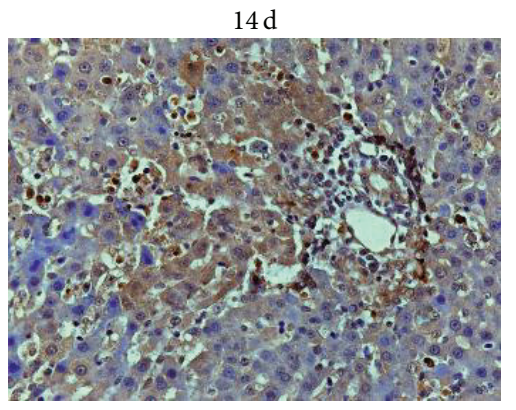
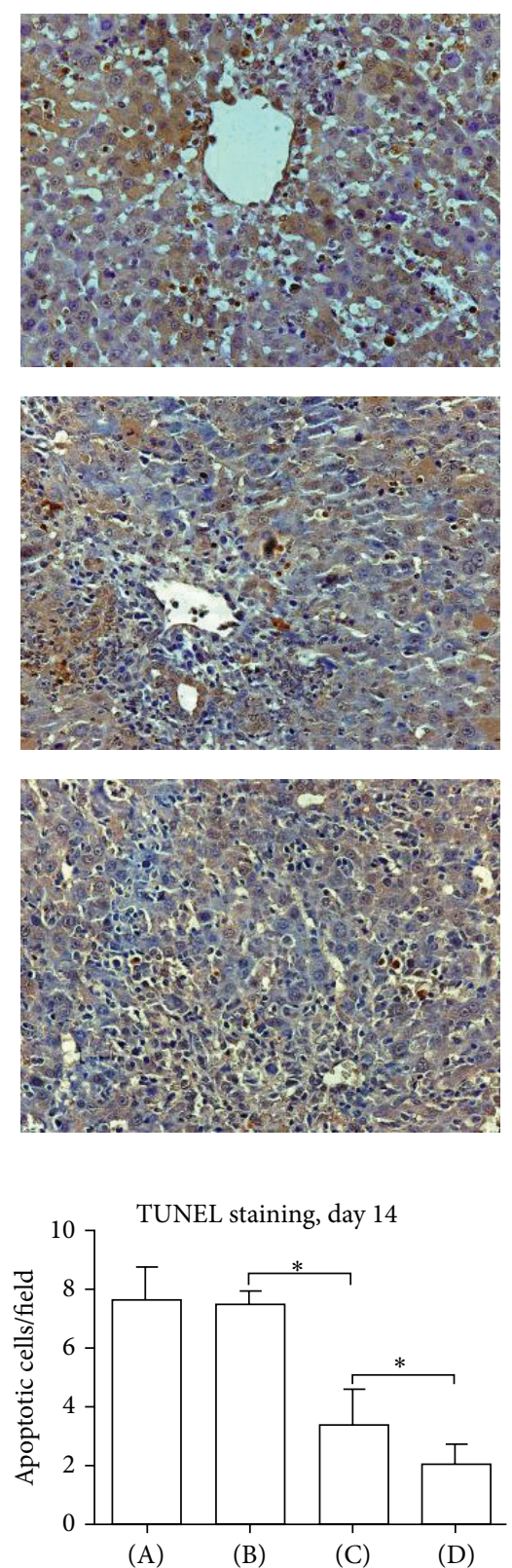

(b)

Figure 4: Apoptotic cells in liver grafts. (A) Normal saline group; (B) HO-1 group; (C) BMMSCs group; (D) HO-1/MSCs group. (a) Histological sections from rats of four groups at each time point were subjected to the TUNEL assay (200x). (b) Histogram showing the percentage of positive apoptotic signals in each group on days 1, 7, and 14. Apoptotic cell numbers in the HO-1/MSCs group were significantly lower compared with control groups on POD 1,7 , and 14 , respectively $\left({ }^{*} P<0.05\right)$. 

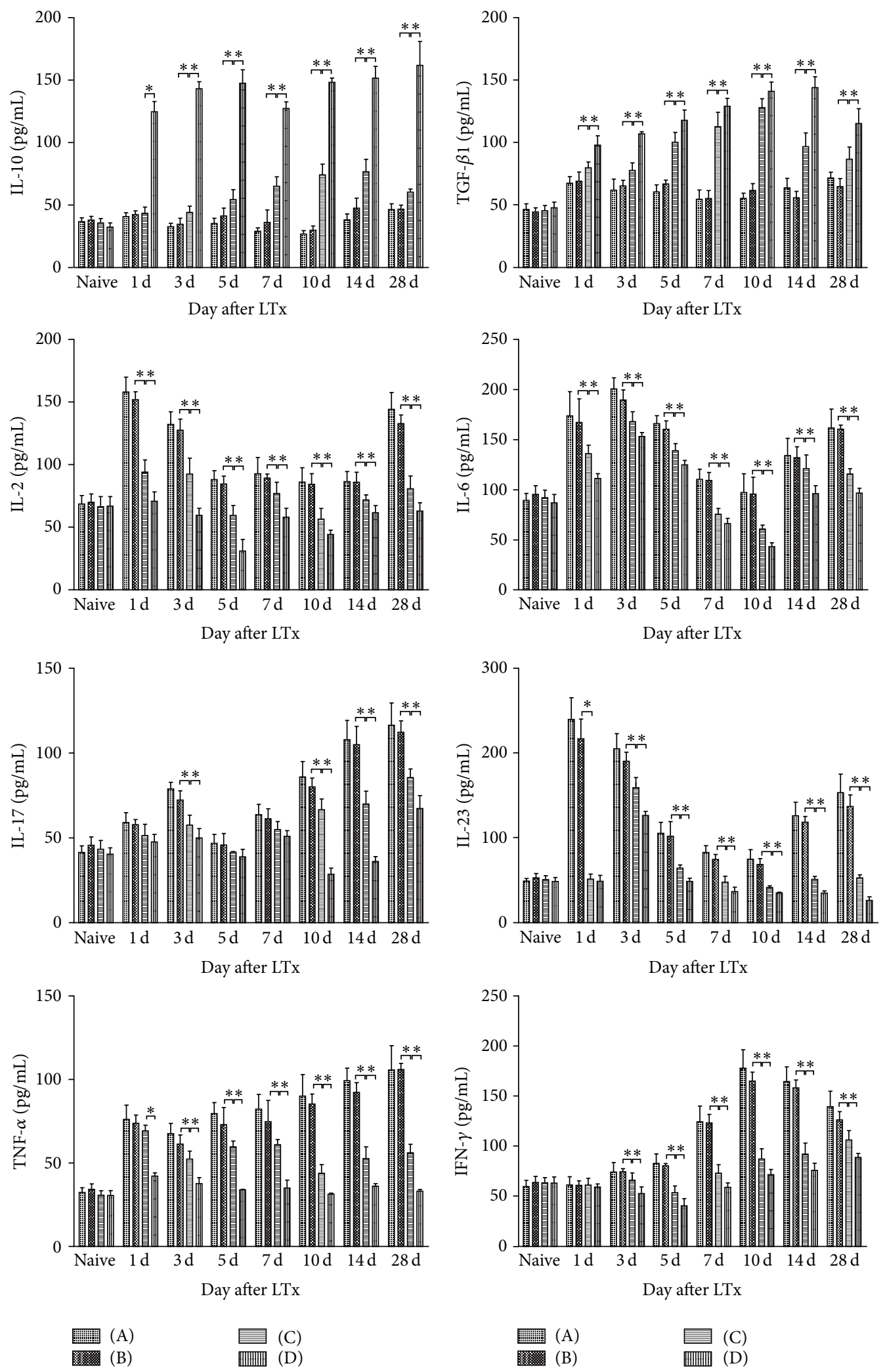

Figure 5: IL-10, TGF- $\beta$, IL-2, IL-6, IL-17, IL-23, TNF- $\alpha$, and IFN- $\gamma$ concentrations in recipient serum. (A) Normal saline group; (B) HO-1 group; (C) BMMSCs group; (D) HO-1/MSCs group. Cytokine concentrations in the recipient serum were measured by ELISA on days 1, 3, 5, $7,10,14$, and 28 after transplantation $\left(n=5\right.$ in each group). Data are expressed as the mean $\pm \mathrm{SD}\left({ }^{*} P<0.05\right)$. Levels of IL- 10 and TGF- $\beta$ were significantly higher, while levels of IL-2, IL-6, IL-17, IL-23, TNF- $\alpha$, and IFN- $\gamma$ were significantly lower in the HO-1/MSCs group than those of control groups. 
$1 \mathrm{~d}$

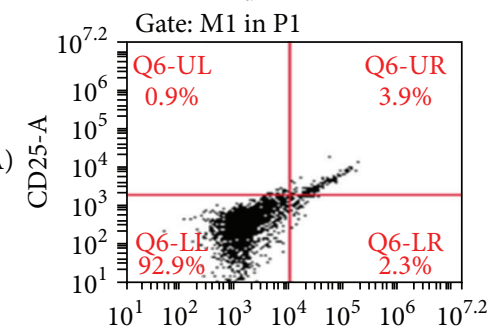

(B)

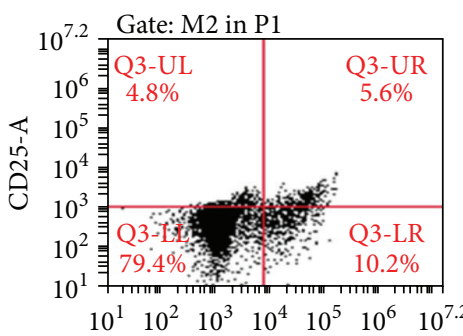

(C)

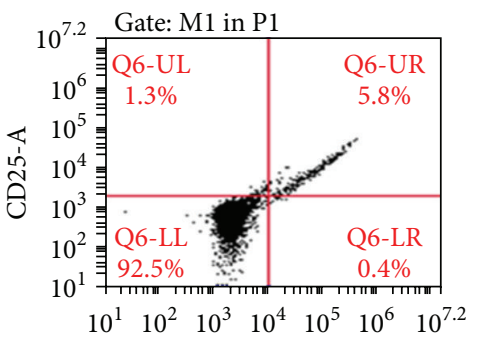

(D)

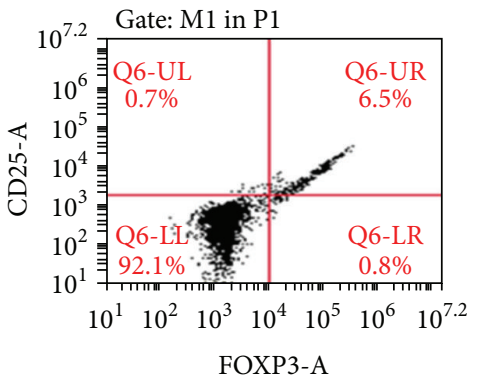

$7 \mathrm{~d}$

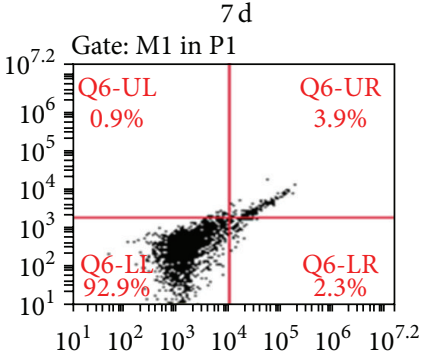

$10^{7.2}$ Gate: M1 in P1

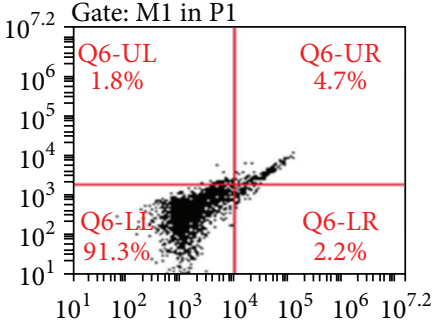

$10^{7.2}$ Gate: M2 in P1

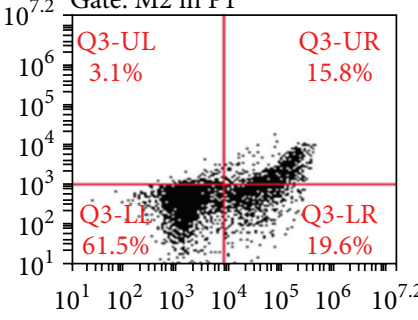$$
\text { Gate: } \mathrm{M} 1 \text { in } \mathrm{P} 1
$$

$14 \mathrm{~d}$

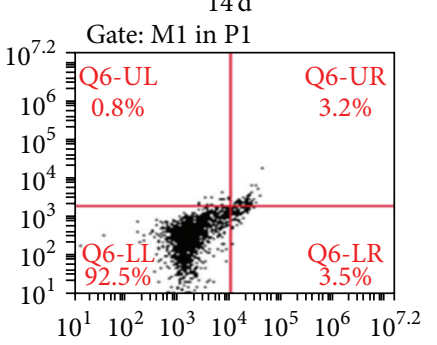

$10^{7.2}$ Gate: $\mathrm{M} 1$ in P1

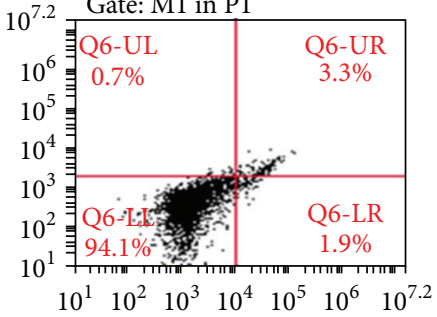

$10^{7.2}$ Gate: $\mathrm{M} 1$ in P1

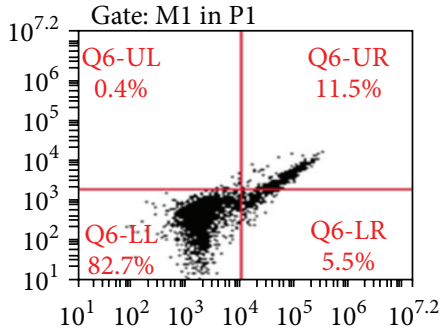

(a)

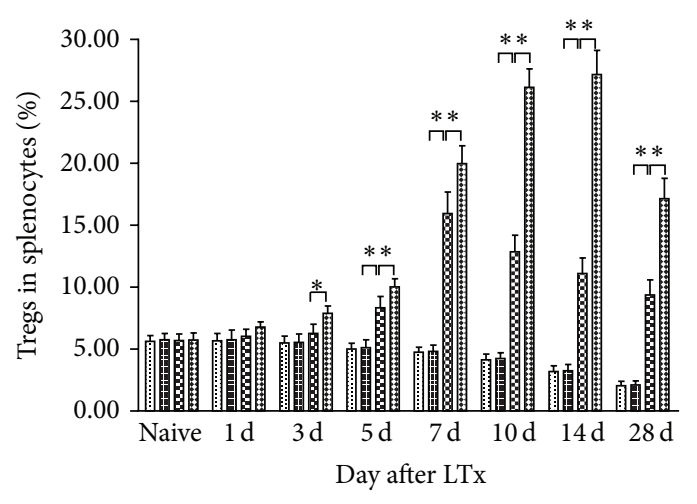
(A)
88 (C)
(B)

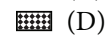

(b)

Figure 6: Proportion of Tregs in recipient splenocytes. (A) Normal saline group; (B) HO-1 group; (C) BMMSCs group; (D) HO-1/MSCs group. Percentages of CD4+CD25+Foxp3+ cells in recipient spleens were measured by flow cytometry. (a) Scatter plots on POD 1, 7, and 14 showing that the percentage of Tregs was higher in HO-1/MSC-treated rats than in the control groups. (b) Histogram showing percentages of Tregs on POD 3, 5, 7, 10,14, and 28. The number of Tregs in the HO-1/MSCs group first increased and then decreased but peaked on POD 14 and were significantly higher than those of other groups on POD $3,5,7,10,14$, and $28\left({ }^{*} P<0.05\right)$. 
immunomodulatory effects. Furthermore, levels of ALT, AST, and TBIL were significantly lower in the HO-1/MSCs group than control groups at all time points. Therefore, these results further confirmed the strong immune regulatory and enhanced protective effects of HO-1/MSCs on liver cells.

The pathogenesis of organ transplant rejection has been shown to be mainly mediated by $\mathrm{T}$ cell immune responses [26]. Functionally, T lymphocytes can be divided into CD4+ Th cells, CD8+ killer T cells (cytotoxic T cells), and suppressor T cells. Th cells can secrete a variety of cytokines, which are classified as Th0, Th1, Th2, Th3, or Th17, to regulate immune responses and play an important role in transplantation immunity [27]. Th1 cells can trigger allogenic transplant rejection by promoting the generation of alloantigen specific cytotoxic T cells and delayed-type hypersensitivity, while Th2 cells exert opposite effects. Therefore, the dynamic Th1/Th2 balance plays an important role in immune tolerance, and the shift from Th1 to Th2 type cells is one mechanism of generating tolerance [28]. As IL-2 and IFN- $\gamma$ are Thl cytokines and IL-10 is a Th2 cytokine, the IL-2/IL-10 ratio closely reflects the rate of rejection and induction of tolerance [29]. The dynamic Th17/Treg balance also is important in immune tolerance. IL6, IL-17, IL-23, and TGF- $\beta$ are Th17/Treg-related cytokines, which determine the transition of T cells into inflammatory Th17 or immune Tregs [30]. As a proinflammatory cytokine, TNF- $\alpha$ is closely related to immunoregulation, cell apoptosis, and $\mathrm{T}$ cell proliferation, and it has been positively correlated with the degree of graft rejection [31]. In this study, levels of IL-10 and TGF- $\beta$ were significantly higher, while those of IL-2, IL-6, IL-17, IL-23, TNF- $\alpha$, and IFN- $\gamma$ were significantly lower, in the HO-1/MSCs group compared with control groups, which further confirmed the strong immune regulatory effects of $\mathrm{HO}-1 / \mathrm{MSCs}$.

Tregs (CD4+CD25+Foxp3+ T cells) exert negative immune regulation and account for approximately $5-10 \%$ of peripheral $\mathrm{CD} 4+\mathrm{T}$ cells. By inhibiting the activation and proliferation of other immune effector cells, Tregs play an important role in the induction of immune tolerance to transplanted tissue. Studies to date have implicated the following mechanisms for Treg-mediated inhibition of other immune effector cells: inhibiting IL-2 production by close contact among cells; preventing antigen presenting cells from expressing stimulus molecules and preventing dendritic cells from maturing; destroying target cells via the granular enzyme/perforin death pathways; consuming local IL-2 to cause loss of stimulatory signals to effector T cells; and inducing production of inhibitory cytokines such as TGF- $\beta$, IL-10, and IL-35 [32]. In this study, the proportion of Tregs was significantly higher in the $\mathrm{HO}-1 / \mathrm{MSC}$ group compared with control groups on POD 3, 5, 7, 10, 14, and 28. Therefore, we concluded that inducing production of Tregs is just one mechanism by which HO-1/MSCs can exert their immunosuppressive effects, and the selective inhibition of Tregs is led by the combined effects of various inhibitory mechanisms.

In summary, in this study, compared with BMMSCs, HO1/MSCs improved the pathology of the transplanted liver, reduce cell apoptosis, improved liver functions, decreased levels of Th1 and Th17 cytokines, and promoted the generation of Tregs. Therefore, we reasoned that HO-1/MSCs could improve the outcome of allogeneic LTx by attenuating the inflammatory response and ACR, with better and more prolonged effects compared to BMMSCs. However, further studies exploring mechanism are needed to fully elucidate how HO-1 helps BMMSCs to attenuate the inflammatory response and ACR. In addition studies using higher doses of HO- 1 are required. We believe that these findings provide a potentially new and effective strategy for suppressing ACR and improving outcomes after LTx.

$\begin{array}{ll}\text { Abbreviations } \\ \text { BMMSCs: } & \text { Bone marrow mesenchymal stem cells } \\ \text { BN: } & \text { Brown Norway } \\ \text { ELISA: } & \text { Enzyme-linked immunosorbent assay } \\ \text { GFP: } & \text { Green fluorescent protein } \\ \text { HO-1: } & \text { Heme oxygenase-1 } \\ \text { LTx: } & \text { Liver transplantation } \\ \text { ACR: } & \text { Acute cellular rejection } \\ \text { POD: } & \text { Postoperative days } \\ \text { IFN- } \gamma: & \text { Interferon- } \gamma \\ \text { IL-2: } & \text { Interleukin-2 } \\ \text { IL-6: } & \text { Interleukin-6 } \\ \text { IL-10: } & \text { Interleukin-10 } \\ \text { IL-17: } & \text { Interleukin-17 } \\ \text { IL-23: } & \text { Interleukin-23 } \\ \text { PBS: } & \text { Phosphate-buffered saline } \\ \text { TGF- } \beta: & \text { Transforming growth factor- } \beta \\ \text { TNF- } \alpha: & \text { Tumor necrosis factor- } \alpha \\ \text { Tregs: } & \text { T regulatory cells } \\ \text { TUNEL: } & \text { Terminal deoxynucleotidyl } \\ & \text { transferase-mediated dUTP nick } \\ & \text { end-labeling. }\end{array}$

\section{Conflict of Interests}

The authors declare no conflict of interests.

\section{Authors' Contribution}

Bin $\mathrm{Wu}$ participated in performance of the research, data analysis, and writing of the paper. Hong-Li Song participated in research design, data analysis, and writing of the paper. Yang Yang, Ming-Li Yin, Bo-Ya Zhang, and Yi Cao participated in performance of the research. Chong Dong participated in research design. Zhong-Yang Shen participated in research design, data analysis, and writing of the paper. All authors have read and approved the final paper.

\section{Acknowledgments}

This work was supported by grants from the National Natural Science Foundation of China (nos. 81270528 and 81441022), the National Science Foundation of Tianjin, China (nos. 08JCYBJC08400, 11JCZDJC27800, and 12JCZDJC25200), and the Technology Foundation of Health Bureau in Tianjin, China (no. 2011KY11). The authors thank the Key Laboratory of Emergency and Care Medicine of Ministry of Health and 
the Tianjin Key Laboratory of Organ Transplantation for allowing this work to progress in their laboratories.

\section{References}

[1] M. Desai and J. Neuberger, "Chronic liver allograft dysfunction," Transplantation Proceedings, vol. 41, no. 2, pp. 773-776, 2009.

[2] M. J. Weiss, C. Y. Ng, and J. C. Madsen, "Tolerance, xenotransplantation: future therapies," Surgical Clinics of North America, vol. 86, no. 5, pp. 1277-1296, 2006.

[3] A. Bertolo, T. Thiede, N. Aebli, M. Baur, S. J. Ferguson, and J. V. Stoyanov, "Human mesenchymal stem cell co-culture modulates the immunological properties of human intervertebral disc tissue fragments in vitro," European Spine Journal, vol. 20, no. 4, pp. 592-603, 2011.

[4] M. Barone, M. P. Scavo, E. Maiorano, A. Di Leo, and A. Francavilla, "Bone marrow-derived stem cells and hepatocarcinogenesis in hepatitis B virus transgenic mice," Digestive and Liver Disease, vol. 46, no. 3, pp. 243-250, 2014.

[5] M. Muraca, "Evolving concepts in cell therapy of liver disease and current clinical perspectives," Digestive and Liver Disease, vol. 43, no. 3, pp. 180-187, 2011.

[6] Y.-R. Kuo, C.-C. Chen, S. Goto, P.-Y. Lin, F.-C. Wei, and C.-L. Chen, "Mesenchymal stem cells as immunomodulators in a vascularized composite allotransplantation," Clinical and Developmental Immunology, vol. 2012, Article ID 854846, 8 pages, 2012.

[7] J. L. Reading, S. Sabbah, S. Busch, and T. I. M. Tree, "Mesenchymal stromal cells as a means of controlling pathological T-cell responses in allogeneic islet transplantation," Current Opinion in Organ Transplantation, vol. 18, no. 1, pp. 59-64, 2013.

[8] M. M. Duffy, J. Pindjakova, S. A. Hanley et al., "Mesenchymal stem cell inhibition of T-helper 17 cell-differentiation is triggered by cell-cell contact and mediated by prostaglandin E2 via the EP4 receptor," European Journal of Immunology, vol. 41, no. 10, pp. 2840-2851, 2011.

[9] H. Song, K. Kwon, S. Lim et al., "Transfection of mesenchymal stem cells with the FGF-2 gene improves their survival under hypoxic conditions," Molecules and Cells, vol. 19, no. 3, pp. 402407, 2005.

[10] N. G. Abraham and A. Kappas, "Pharmacological and clinical aspects of heme oxygenase," Pharmacological Reviews, vol. 60, no. 1, pp. 79-127, 2008.

[11] S. Brouard, L. E. Otterbein, J. Anrather et al., "Carbon monoxide generated by heme oxygenase 1 suppresses endothelial cell apoptosis," Journal of Experimental Medicine, vol. 192, no. 7, pp. 1015-1025, 2000.

[12] K. Sodhi, N. Puri, G. Favero et al., "Fructose mediated nonalcoholic fatty liver is attenuated by HO-1-SIRT1 module in murine hepatocytes and mice fed a high fructose diet," PLoS ONE, vol. 10, no. 6, Article ID e0128648, 2015.

[13] T. Tsubokawa, K. Yagi, C. Nakanishi et al., "Impact of antiapoptotic and anti-oxidative effects of bone marrow mesenchymal stem cells with transient overexpression of heme oxygenase-1 on myocardial ischemia," The American Journal of Physiology-Heart and Circulatory Physiology, vol. 298, no. 5, pp. H1320-H1329, 2010.

[14] A. Zarjou, J. Kim, A. M. Traylor et al., "Paracrine effects of mesenchymal stem cells in cisplatin-induced renal injury require heme oxygenase-1," American Journal of PhysiologyRenal Physiology, vol. 300, no. 1, pp. F254-F262, 2011.

[15] J.-G. Li, Y.-X. Zhuan-sun, B. Wen et al., "Human mesenchymal stem cells elevate CD4+CD25+CD127low/- regulatory T cells of asthmatic patients via heme oxygenase-1," Iranian Journal of Allergy, Asthma and Immunology, vol. 12, no. 3, pp. 228-235, 2013.

[16] K. Yamashita, R. Öllinger, J. McDaid et al., "Heme oxygenase-1 is essential for and promotes tolerance to transplanted organs," The FASEB Journal, vol. 20, no. 6, pp. 776-778, 2006.

[17] C. P. Hodgkinson, J. A. Gomez, M. Mirotsou, and V. J. Dzau, "Genetic engineering of mesenchymal stem cells and its application in human disease therapy," Human Gene Therapy, vol. 21, no. 11, pp. 1513-1526, 2010.

[18] L. Vanella, D. H. Kim, D. Asprinio et al., "HO-1 expression increases mesenchymal stem cell-derived osteoblasts but decreases adipocyte lineage," Bone, vol. 46, no. 1, pp. 236-243, 2010.

[19] D. Chabannes, M. Hill, E. Merieau et al., "A role for heme oxygenase-1 in the immunosuppressive effect of adult rat and human mesenchymal stem cells," Blood, vol. 110, no. 10, pp. 3691-3694, 2007.

[20] N.-N. Fu, H.-L. Song, B.-J. Wu, and T. Liu, "Effects of HO-1 modified rat bone marrow mesenchymal stem cells on lymphocytes in vitro," Chinese Journal of Microbiology and Immunology, vol. 33, no. 11, pp. 801-806, 2013.

[21] M. F. Pittenger, A. M. Mackay, S. C. Beck et al., "Multilineage potential of adult human mesenchymal stem cells," Science, vol. 284, no. 5411, pp. 143-147, 1999.

[22] N. Kamada and R. Y. Calne, "Orthotopic liver transplantation in the rat. Technique using cuff for portal vein anastomosis and biliary drainage," Transplantation, vol. 28, no. 1, pp. 47-50, 1979.

[23] A. J. Demetris, K. P. Batts, A. P. Dhillon et al., "Banff schema for grading liver allograft rejection: an international consensus document," Hepatology, vol. 25, no. 3, pp. 658-663, 1997.

[24] S. A. Lodhi, K. E. Lamb, and H. U. Meier-Kriesche, "Solid organ allograft survival improvement in the United States: the long-term does not mirror the dramatic short-term success," American Journal of Transplantation, vol. 11, no. 6, pp. 12261235, 2011.

[25] S. Glennie, I. Soeiro, P. J. Dyson, E. W.-F. Lam, and F. Dazzi, "Bone marrow mesenchymal stem cells induce division arrest anergy of activated T cells," Blood, vol. 105, no. 7, pp. 2821-2827, 2005.

[26] K. A. Newell, "Clinical transplantation tolerance," Seminars in Immunopathology, vol. 33, no. 2, pp. 91-104, 2011.

[27] I. N. Crispe, "Hepatic T cells and liver tolerance," Nature Reviews Immunology, vol. 3, no. 1, pp. 51-62, 2003.

[28] O. Alpdogan and M. R. M. van den Brink, "Immune tolerance and transplantation," Seminars in Oncology, vol. 39, no. 6, pp. 629-642, 2012.

[29] J.-F. Feng, F. Chen, H. Liu, and J. Liu, "Induction of immune tolerance by pre-infusion of apoptotic lymphocytes derived from peripheral blood of donor rats before liver transplantation," Minerva Chirurgica, vol. 68, no. 2, pp. 183-189, 2013.

[30] D. Hanidziar and M. Koulmanda, "Inflammation and the balance of Treg and Th17 cells in transplant rejection and tolerance," Current Opinion in Organ Transplantation, vol. 15, no. 4, pp. 411-415, 2010. 
[31] D. Budak, V. T. Yilmaz, H. Akbas et al., "Association between graft function and serum TNF- $\alpha$, TNFR1 and TNFR2 levels in patients with kidney transplantation," Renal Failure, vol. 24, pp. $1-6,2015$.

[32] E. M. Shevach, "Mechanisms of Foxp3 ${ }^{+} \mathrm{T}$ regulatory cell mediated suppression," Immunity, vol. 30, no. 5, pp. 636-645, 2009. 

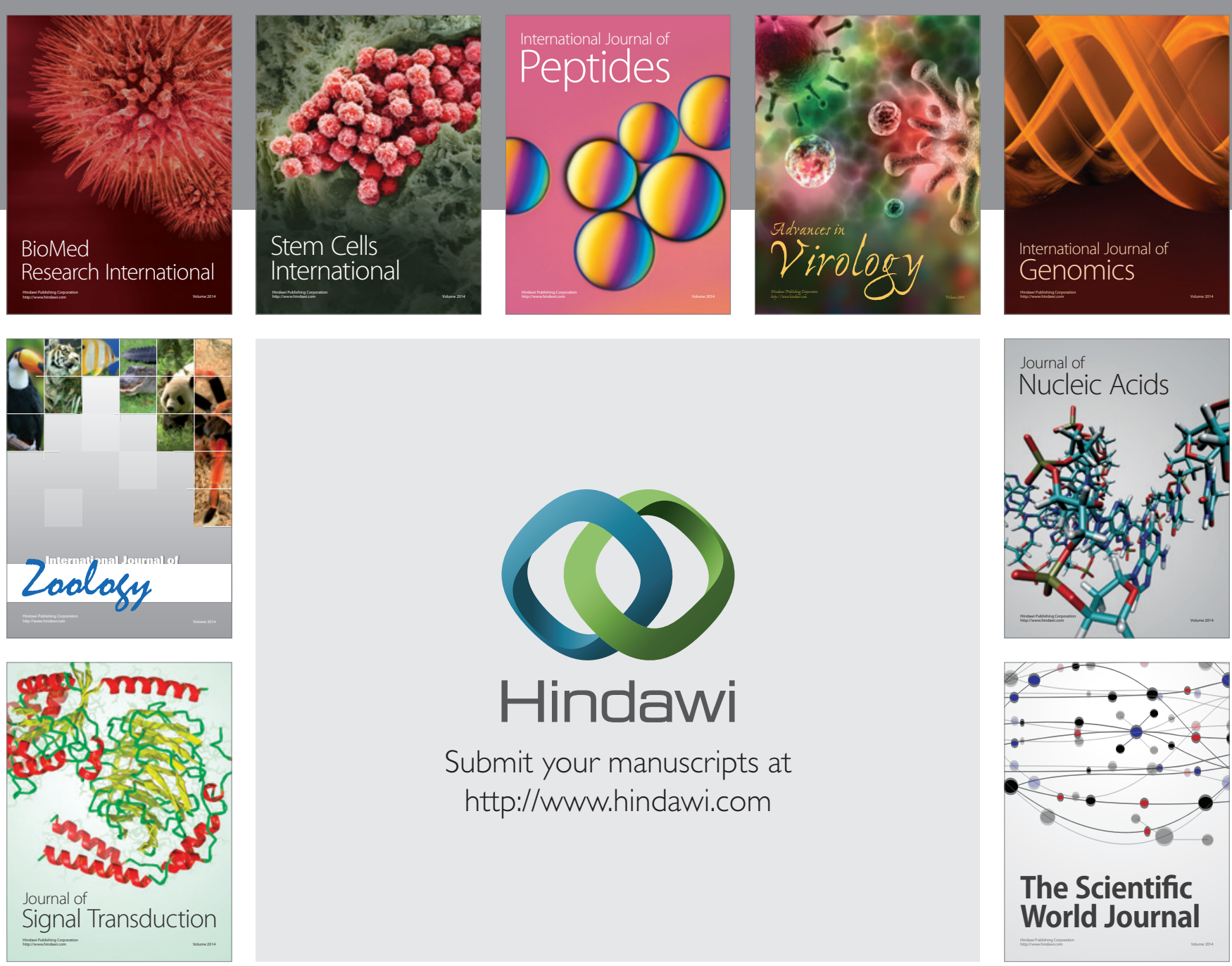

Submit your manuscripts at

http://www.hindawi.com
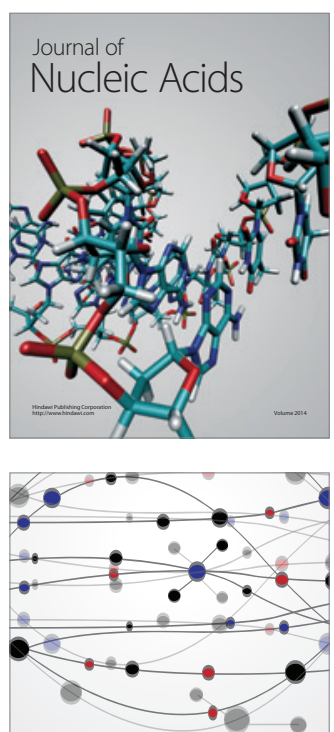

The Scientific World Journal
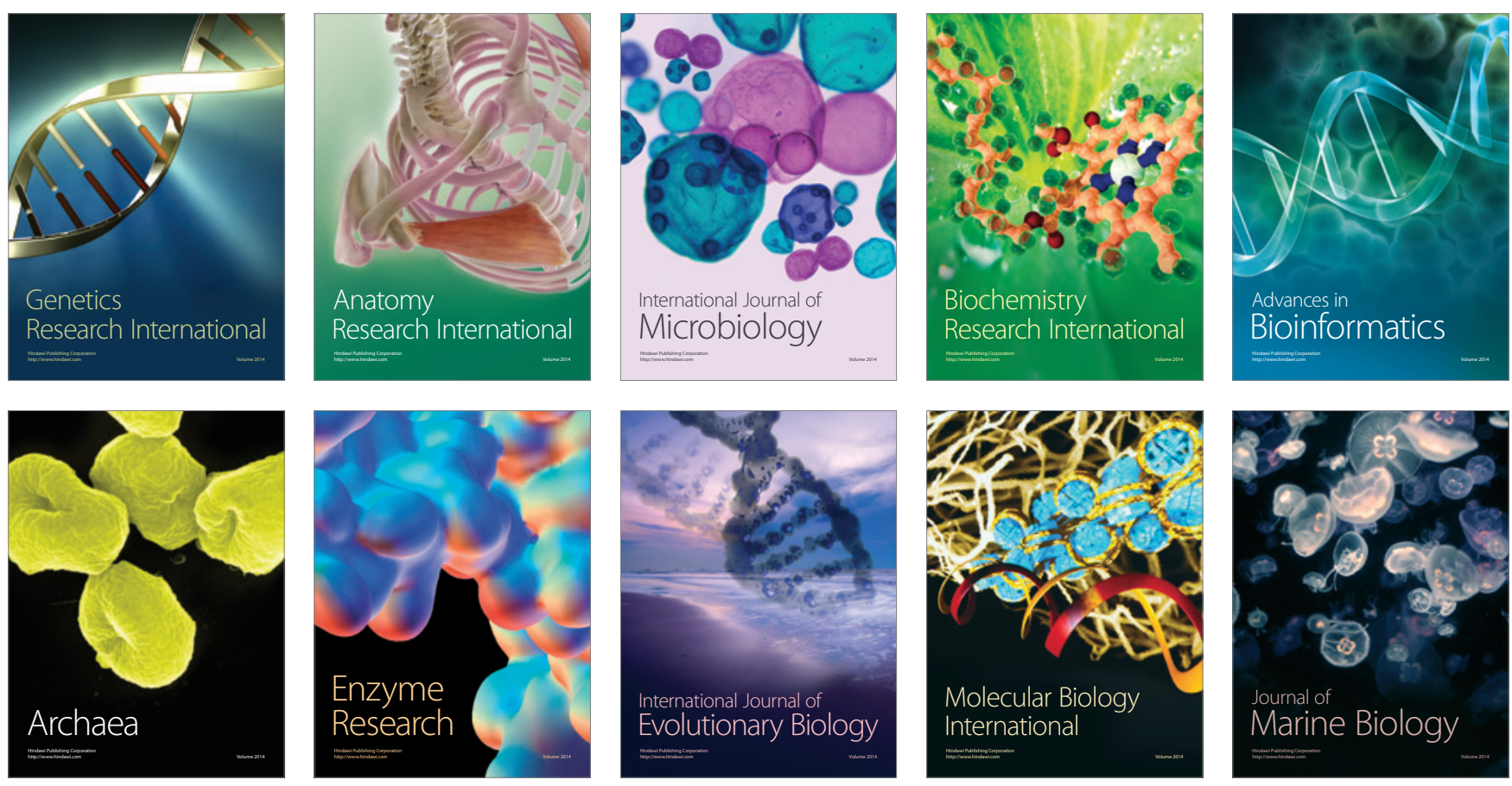